Psychotherapeut 2020 $65: 444-455$ https://doi.org/10.1007/s00278-020-00453-7 Angenommen: 5. August 2020 Online publiziert: 16 . September 2020 (c) Der/die Autor(en) 2020

\begin{abstract}
Neben dem angewandten Verfahren wird der Erfolg einer Psychotherapie maßgeblich auch durch die Person des Therapeuten bestimmt. Vorliegende Studienergebnisse zur Behandlung der sozialen Angststörung berichten keine, durchschnittliche bis hin zu sehr großen Therapeuteneffekten. Im Rahmen einer umfassenden Studie gilt es daher, Varianzen zufälliger Effekte und Effekte soziodemografischer Variablen des Therapeuten zu untersuchen. Des Weiteren ist eine Disaggregation der therapeutischen Beziehung in "Between"(Unterschiede zwischen Therapeutenmittelwerten) und "Within"Anteile (Unterschiede zwischen den Patienten „innerhalb" eines Therapeuten) vorzunehmen. Für die soziale Angststörung liegen bislang keine Studien vor, die insbesondere die Effekte der letztgenannten Parameter untersucht haben.
\end{abstract}

\section{Hintergrund}

Mit einer Lebenszeitprävalenz von $13 \%$ (Kessler et al. 2012) gehört die soziale Angststörung zu den häufigsten psychischen Störungen. Sie geht mit starken psychosozialen Beeinträchtigungen einher und ist mit hohen sozioökonomischen Kosten verbunden (Kessler et al. 2012). Effektive Behandlungen sind pharmakologische Therapien sowie

Uwe Altmann ${ }^{1}$ - Brian Schwartz ${ }^{2}$. Desiree Schönherr' ${ }^{1}$ Julian Rubel ${ }^{3}$. Ulrich Stangier ${ }^{4} \cdot$ Wolfgang Lutz $^{2} \cdot$ Bernhard Strauß ${ }^{1}$

${ }^{1}$ Institut für Psychosoziale Medizin, Psychotherapie und Psychoonkologie, Universitätsklinikum Jena, Jena, Deutschland

${ }^{2}$ Klinische Psychologie und Psychotherapie, Universität Trier, Trier, Deutschland

${ }^{3}$ Fachgebiet Psychologie, Justus-Liebig-Universität, Gießen, Deutschland

${ }^{4}$ Klinische Psychologie und Psychotherapie, Goethe-Universität, Frankfurt am Main, Deutschland

\title{
Therapeuteneffekte bei der ambulanten Behandlung sozialer Ängste
}

psychologische Therapien im Einzelund im Gruppensetting (Barkowski et al. 2016; Mayo-Wilson et al. 2014). Aktuelle Vergleichsstudien legen nahe, dass verschiedene Therapieverfahren in etwa die gleiche Effektivität aufweisen: Leichsenring et al. (2013) verglichen Verhaltenstherapie (VT) und tiefenpsychologische Therapie (TP) und berichteten eine bessere Prä-post-Symptomreduktion zugunsten der VT im Einzelsetting. Im Hinblick auf Katamnesewerte stellten Leichsenring et al. (2014) jedoch keine Unterschiede zwischen VT und TP fest. Bögels et al. (2014) fanden keine Unterschiede zwischen VT und TP bezüglich der Remissionsraten. Bei einigen dimensionalen Outcomes zeigte die TP eine bessere Prä-post-Symptomreduktion. In der Studie von Bohn et al. (2013) fanden sich keine Unterschiede zwischen VT und TP, sowohl was das Outcome betrifft als auch die Häufigkeit und Stärke früher Symptomveränderungen („sudden gain“). Desgleichen stellten Hunger et al. (2018) keine Outcome-Unterschiede bei VT und systemischer Psychotherapie sozialer Ängste fest. In der Studie von Herbert et al. (2009) fanden sich keine Unterschiede in der Symptomatik nach Behandlungsende zwischen VT im Gruppensetting, VT im Einzelsetting und supportiver Therapie im Einzelsetting. Bei verhaltensbezogenen Outcomes zeigten sich jedoch bessere Werte in beiden VT Bedingungen.
Neben dem Therapieverfahren sind die Gründe für einen Therapieerfolg aber auch in der Person des Therapeuten zu suchen. Die Forschung zu Therapeuteneffekten (z.B. Baldwin und Imel 2013; Crits-Christoph et al. 1991; Johns et al. 2019) hat empirisch belegt, dass es Therapeuten mit besonders hohen („super shrinks"), durchschnittlichen und besonders geringen Erfolgsquoten („pseudo shrinks“) gibt. Im Durchschnitt über mehrere Studien hinweg können etwa 5\% (Baldwin und Imel 2013; Johns et al. 2019) bis 8,6\% (CritsChristoph et al. 1991) der Varianz des Therapieergebnisses durch Therapeuteneffekte erklärt werden. Studien zur Behandlung der sozialen Angststörung berichten keine (Almlöv et al. 2010; Altmann et al. 2020a), gemessen an den obigen Metaanalysen durchschnittliche (5-7\% bei verschiedenen Angstskalen; Leichsenring et al. 2013) bis hin zu sehr großen Therapeuteneffekten (23 bzw. $30 \%$ bei Depressivität und interpersonalen Problemen bei Altmann et al. 2020b).

Die Größe des Therapeuteneffekts ist vom therapeutischen Setting, von Therapeuten-, Patienteneigenschaften und vom verwendeten Ergebnismaß abhängig. In der Metaanalyse von Baldwin and Imel (2013) waren Therapeuteneffekte in manualisierten Therapien kleiner als in Therapien unter naturalistischen Bedingungen ( $3 \%$ vs. $7 \%$ ). Hingegen erklärten Therapeuteneffekte in der spä- 
teren Metaanalyse von Johns et al. (2019) mehr Ergebnisvarianz in randomisierten kontrollierten Studien (RCT) als in naturalistischen Studien (8,2\% vs. $5 \%$ ). Ihren kontraintuitiven Befund erklärten Johns et al. (2019) mit der Heterogenität der untersuchten RCT und deren vergleichsweise geringen Stichprobenumfängen. Bezüglich des therapeutischen Settings berichteten Spitzer et al. (2015), dass bei stationären Psychotherapien mit gruppentherapeutischem Schwerpunkt keine substanziellen Effekte des Bezugstherapeuten auf das symptombezogene Behandlungsergebnis vorlagen. Sie erklärten dies damit, dass Patienten im von ihnen untersuchten Setting mehrere und zudem wechselnde Bezugstherapeuten hatten.

Im Hinblick auf Patienteneigenschaften berichteten Johns et al. (2019), dass Therapeuteneffekte bei Patienten mit geringer Symptomatik kaum und bei Patienten mit starker initialer Symptomatik stark ausgeprägt waren. Darüber hinaus zeigten Huppert et al. (2001) und Lutz et al. (2007), dass die Therapeuteneffekte vom Outcome abhängig sind. Lutz et al. (2007) berichteten z. B. einen Therapeuteneffekt von $8 \%$ für dimensionale Ergebnismaße und von $17 \%$ für Remissionsraten.

Therapeutenvariablen wie Alter, Geschlecht, Qualifikationsgrad und theoretische Ausrichtung waren in den Studien von Huppert et al. (2001) und Chow et al. (2015) nicht prädiktiv für das Therapieergebnis. Die Berufserfahrung des Therapeuten hatte zumindest bei einigen Ergebnismaßen einen Effekt (Huppert et al. 2001). Des Weiteren konnten Persönlichkeitseigenschaften des Therapeuten das Therapieergebnis vorhersagen: Verhaltenstherapeuten mit unterdurchschnittlichen Werten auf der Skala Offenheit für neue Erfahrungen hatten in derStudie von Delgadillo et al. (2020) bessere Therapieergebnisse als Therapeuten mit hohen Werten auf der Skala Offenheit für neue Erfahrungen. Therapeuten mit überdurchschnittlich guten Therapieergebnissen gelingt es zudem, Patienten länger in der Therapie zu halten (Saxon et al. 2017). Sie haben überdurchschnittlich gute interpersonelle Fähigkeiten, die insbesondere in schwierigen Therapiesi- tuationen relevant sind (z. B. emotionale Kontrolle und Empathie; Heinonen und Nissen-Lie 2020; Rief et al. 2020).

Im Hinblick auf die Untersuchung von Therapeuteneffekten lassen sich drei methodische Ansätze unterscheiden: Zum einen können Therapeuteneffekte bei sog. genesteten Daten (mehrere Patienten werden durch einen Therapeuten behandelt) mithilfe der Intra-KlassenKorrelation (ICC) von zufälligen Effekten quantifiziert werden („random effects studies“; Baldwin und Imel 2013). Allerdings sind zufällige Effekte unspezifisch - es kann nicht gesagt werden, was genau einen Therapeuten erfolgreich oder weniger erfolgreich macht. Der zweite Ansatz untersucht spezifische Therapeutenvariablen, wie Alter, Geschlecht, Erfahrung oder Persönlichkeitseigenschaften des Therapeuten („fixed effects studies“; Baldwin und Imel 2013). Der dritte Ansatz trägt dem Umstand Rechnung, dass Psychotherapie dyadischer Natur ist und der Therapieprozess dementsprechend gleichzeitig von Therapeut und Patient beeinflusst wird (Hara et al. 2017). Hierbei werden Prozessvariablen in einen Therapeutenund einen Patientenanteil disaggregiert (Constantino et al. 2017). Hara et al. (2017) untersuchten mit diesem Ansatz z. B. Zusammenhänge zwischen der Empathie des Therapeuten, der Compliance bezüglich der Hausaufgaben und dem Therapieergebnis. Es zeigte sich, dass die „Within-therapist-Anteile“ der Empathie (d.h. die Unterschiede zwischen den Patienten „innerhalb“ eines Therapeuten) mit der Compliance bezüglich der Hausaufgaben assoziiert waren. Die „Between-therapist-Anteile“ der Empathie (d.h. die Unterschiede zwischen den Therapeutenmittelwerten) sagten hingegen eine Reduktion der Ängste vorher.

$\mathrm{Zu}$ den prozessrelevanten Variablen, denen sowohl ein Patienten- als auch ein Therapeutenanteil inhärent ist, zählen u.a. die therapeutische Beziehung und die nonverbale Synchronisation während der Therapiesitzungen. Die therapeutische Beziehung wird als allgemeiner Wirkfaktor der Psychotherapie aufgefasst (Ackerman and Hilsenroth 2003), da sie unabhängig von psychischer Stö- rung und Art der Psychotherapie mit dem Therapieergebnis assoziiert ist (Del Re et al. 2012; Flückiger et al. 2012, 2018; Horvath et al. 2011). Sie umfasst sowohl Therapeutenanteile wie Empathie als auch Patientenanteile wie Vertrauen (Ackerman und Hilsenroth 2003). Die Befunde zur therapeutischen Beziehung bei der Behandlung der sozialen Angststörung sind allerdings heterogen. Einige Studien berichten einen signifikanten Zusammenhang zwischen therapeutischer Beziehung und Therapieergebnis (z.B. Altmann et al. 2020a; Haug et al. 2016), andere Autoren fanden hingegen keinen (z.B. Andersson et al. 2012; Mörtberg 2014; Woody and Adessky 2002).

Weiterhin liegen gemäß dem Kenntnisstand der Autoren des vorliegenden Beitrags für die soziale Angststörung keine Studien vor, die Effekte der Withinund Between-therapist-Anteile der therapeutischen Beziehung untersuchten. Für eine störungsheterogene Stichprobe (Baldwin et al. 2007) und eine Stichprobe depressiver Patienten (Zuroff et al. 2010) wird zumindest berichtet, dass die Between-therapist-Anteile der therapeutischen Beziehung eine Symptomreduktion vorhersagten. Die Withintherapist-Anteile waren hingegen nur in der depressiven Stichprobe signifikant. Baldwin et al. (2007) schlussfolgern deshalb, dass patienten- und therapeutenseitige Anteile an der Varianz von Beziehung-Ratings der Patienten nicht gleichermaßen das Therapieergebnis vorhersagen und deshalb möglichst getrennt betrachtet werden sollten.

Eine weitere prozessrelevante sowie in einen Patienten- und einen Therapeutenanteil disaggregierbare Variable ist die nonverbale Synchronisation von Patient und Therapeut während der Therapiesitzung. Unter dem Begriff nonverbale Synchronisation werden kurzzeitige Phänomene wie das Imitieren der Mimik, das Angleichen der Stimme und die gleichzeitige Ausführung ähnlicher Körperbewegungen zusammengefasst (Altmann 2013). Sie ist für die emotionale und soziale Entwicklung von Kindern bedeutsam (Feldman 2012) und bei Erwachsenen mit prosozialen Verhaltensweisen, wahrgenommener sozialer 
Psychotherapeut 2020 -65:444-455 https://doi.org/10.1007/s00278-020-00453-7

(c) Der/die Autor(en) 2020

U. Altmann · B. Schwartz · D. Schönherr · J. Rubel · U. Stangier · W. Lutz · B. Strauß

Therapeuteneffekte bei der ambulanten Behandlung sozialer Ängste

\section{Zusammenfassung}

Hintergrund. Die Studie untersuchte Therapeuteneffekte bei der Psychotherapie der sozialen Angststörung unter Verwendung von 3 methodischen Ansätzen: 1. Varianzen zufälliger Effekte, 2. Effekte soziodemografischer Variablen und 3. eine Disaggregation von therapeutischer Beziehung und nonverbaler Synchronisation in einen "Between"- und einen "Within"-Anteil der Therapeutenvarianz sowie deren Vorhersage des Outcome.

Material und Methoden. Die Patienten wurden mit manualisierter supportiverexpressiver Therapie $(n=54)$, manualisierter kognitiver Verhaltenstherapie $(n=102)$ oder naturalistischer kognitiver Verhaltenstherapie ( $n=111$ ) behandelt (insgesamt $n=267$ ). Es wurden Patientenbeurteilungen interpersonaler Probleme (Inventar zur Erfassung interpersonaler Probleme, IIP-32), von
Depressionssymptomen (Beck-DepressionsInventar, BDI) und der therapeutischen Beziehung (Helping Alliance Questionnaire, $H A Q)$ erfasst. Die nonverbale Synchronisation wurde mithilfe der Motion Energy Analysis und zeitreihenanalytischer Verfahren quantifiziert. Die Zusammenhangsanalysen erfolgten mit Mehrebenenmodellen. Ergebnisse. Alter und Geschlecht des Therapeuten waren nicht prädiktiv für die zum Therapieende gemessenen IIP32- und BDI-Werte. Therapeuteneffekte in Form zufälliger Effekte (Intra-KlassenKorrelation $[$ ICC] $=23,4 \%$ bzw. 30,1\%) erklärten ein Vielfaches mehr an OutcomeVarianz als das Therapieverfahren (ICC $=6 \%$ bzw. ICC $=1,6 \%$ ). Bei der nonverbalen Synchronisation waren Between-therapistund Within-therapist-Anteil mit der Reduktion des IIP-32 assoziiert. Der Between-therapistAnteil des HAQ sagte eine Reduktion des BDI vorher.

Schlussfolgerung. Bei der Behandlung sozialer Ängste sind Therapeuteneffekte bedeutsamer als das Therapieverfahren. Desgleichen waren die Therapeutenanteile bei den prozessrelevanten Variablen therapeutische Beziehung und nonverbale Synchronisation bedeutsamer als die patienten- bzw. dyadenspezifischen Anteile.

Schlüsselwörter

Interpersonale Beziehungen · Rolle des Behandelnden - Therapeutische Beziehung - Nonverbale Synchronisation . Behandlungsergebnis

\section{Therapist effects in outpatient treatment of social anxiety disorder}

\section{Abstract}

Background. The study examined therapist effects in the psychotherapy of social anxiety disorder using three methodological approaches: 1 ) variances of random effects, 2) effects of sociodemographic therapist variables and 3 ) disaggregation of therapeutic relationship and movement synchrony into between-therapist and within-therapist components.

Material and methods. The patients $(n=267)$ were treated either with manually guided supportive-expressive therapy $(n=54)$, manually guided cognitive behavior therapy $(n=102)$ or naturalistic cognitive behavior therapy $(n=111)$. Patient self-reports of interpersonal problems (inventory of interpersonal problems, IIP-32), depression
(Beck depression inventory, BDI) at the end of treatment and ratings of the therapeutic alliance (helping alliance questionnaire, $\mathrm{HAQ}$ ) were collected. Movement synchrony was quantified by means of motion energy analysis and time series analytical procedures. The data analyses were carried out with multilevel models.

Results. The age and sex of the therapist were not predictive for IIP-32 and BDI values at the end of therapy. Therapist effects modelled as random effects (intraclass correlation, ICC $=23.4 \%$ and $30.1 \%$, respectively) explained much more outcome variance than the therapeutic approach (ICC $=6 \%$ and ICC $=1.6 \%$, respectively). With respect to movement synchrony, the between-therapist and within-therapist parts were associated with a reduction of the IIP-32 scores. The between-therapist variance of the $\mathrm{HAQ}$ was predictive for a reduction of the $\mathrm{BDI}$.

Conclusion. In the psychotherapy of social anxiety disorder, therapist effects are more important than the therapeutic procedure. Similarly, the therapist part of the processrelevant variables therapeutic alliance and movement synchrony are more important than the patient or dyad-specific part.

\section{Keywords}

Interpersonal relations - Professional role . Therapeutic alliance $\cdot$ Movement synchrony . Treatment outcome
Bindung, sozialen Kognitionen und positivem Affekt assoziiert (Mogan et al. 2017). Aktuelle Psychotherapiestudien zeigten, dass häufige Bewegungssynchronisation („movement synchrony“, z. B. gleichzeitiges Verändern der Körperhaltung oder synchrones Nicken) mit einem geringeren Risiko für Therapieabbrüche (Paulick et al. 2018; Schoenherr et al. 2019b), einer besseren therapeutischen Allianz (Wiltshire et al. 2020), einer Reduktion interpersoneller Pro- bleme (Altmann et al. 2020b; Ramseyer und Tschacher 2011) und einer Reduktion von Bindungsängsten (Schoenherr et al. 2019d) assoziiert ist. Neben der Häufigkeit der Synchronisation ist auch die „Drive-driven“-Rolle therapeutisch bedeutsam. Eine bessere therapeutische Beziehung (Ramseyer und Tschacher 2011) und ein besseres Therapieergebnis (Altmann et al. 2020b) sind $\mathrm{zu}$ erwarten, wenn der Therapeut häufiger der Initiator der Synchronisation (bzw. der „Drive“) ist und Patienten dementsprechend häufiger den Therapeuten nonverbal imitieren (bzw. der „Driven“ sind). Allerdings sank das Abbruchrisiko, wenn in der Frühphase der Therapie häufiger der Patient der Drive während des Synchronisierens war (Schoenherr et al. 2019b). Das „In-Sync-Therapiemodell“" von Koole und Tschacher (2016) versteht nonverbale Synchronisation als Indikator der therapeutischen Allianz und wesentlichen Einflussfaktor auf die 
(maladaptive) Emotionsregulation der Patienten. Hingegen ist ein Kondensat des Reviews von Wiltshire et al. (2020) die Frage, ob therapeutische Beziehung und nonverbale Synchronisation auf ein gemeinsames übergeordnetes Konstrukt referieren oder nonverbale Synchronisation eher als ein eigenständiges therapierelevantes Konstrukt zu sehen ist. Da das In-Sync-Therapiemodell hauptsächlich auf der Studie von Ramseyer und Tschacher (2011) basiert, ist eine weitere empirische Fundierung gefordert worden, z.B. welchen therapeutischen Effekt nonverbale Synchronisation hat, wenn sie willentlich herbeigeführt wird, oder welche Rolle Therapeutenmerkmale spielen (Altmann et al. 2020b). Des Weiteren ist unklar, ob für die Vorhersage des Therapie-Outcome eher der Therapeutenanteil (der Between-therapist-Anteil im Sinne eines „trait“), der Patientenanteil (der Between-patientAnteil im Sinne eines Trait) oder eher der Situationsanteil (der Within-therapist-Anteil im Sinne eines „state“) der nonverbalen Synchronisation relevant ist.

\section{Fragestellung}

Die vorliegende Studie untersucht Therapeuteneffekte bei der ambulanten psychotherapeutischen Behandlung der sozialen Angststörung und nutzt alle drei der oben genannten methodischen Ansätze: Zum einen wird der Effekt der Therapeutenvariablen Alter und Geschlecht untersucht. Nach Huppert et al. (2001) und Chow et al. (2015) ist bezüglich dieser Variablen kein $\mathrm{Zu}$ sammenhang mit dem Therapieergebnis anzunehmen. Zum zweiten wurden „unspezifische“ Therapeuteneffekte anhand von Mehrebenenmodellen und zufälligen Effekten untersucht. Gemäß der aktuellen Metaanalyse von Johns et al. (2019) wurde eine ICC von $5 \%$ erwartet. Zum dritten wurden die Prozessvariablen therapeutische Beziehung und nonverbale Synchronisation in ihre Within- und Between-therapist-Anteile disaggregiert. In Anlehnung an Baldwin et al. (2007) und Hara et al. (2017) wurde angenommen, dass nur deren Between-therapist-
Anteile mit einer Symptomreduktion assoziiert sind.

\section{Methoden}

\section{Hintergrund}

Die vorliegende Untersuchung ist eine Sekundäranalyse der Daten des Projekts Timing nonverbaler Patient-TherapeutInteraktionen und Therapieerfolg bei sozialen Phobien (TIMPATHIN; Altmann et al. 2020b; Schoenherr et al. 2019b, 2019d). Diese bizentrische Studie (Jena, Trier) untersuchte Patienten mit sozialer Angststörung, die entweder mithilfe manualisierter Verhaltenstherapie (MANVT), manualisierter tiefenpsychologisch fundierter Psychotherapie (MAN-TP) oder mit Verhaltenstherapie unter naturalistischen Bedingungen (NAT-VT) behandelt wurden. Die manualisierten Therapien wurden im Rahmen des multizentrischen SOPHO-NET Trial (Leichsenring et al. 2013, 2014) durchgeführt und bildeten u. a. die Basis für ein bindungsorientiertes Teilprojekt in Jena (z. B. Manes et al. 2016). Bislang konnte gezeigt werden, dass häufige Bewegungssynchronisation in den frühen Therapiesitzungen verschiedene Indikatoren des Therapieerfolgs vorhersagt (Altmann et al. 2020b; Schoenherr et al. 2019b, 2019d). Die vorliegende Untersuchung grenzt sich von den bisherigen Studien insbesondere dadurch $\mathrm{ab}$, dass erstmals prozessrelevante Variablen in Between- und Within-Therapeutenanteile disaggregiert und deren Effekte separat untersucht werden. Weiterhin soll darauf hingewiesen werden, dass Unterschiede zwischen den Therapieverfahren nicht in den Blick genommen werden; diesbezüglich wird auf die Studien von Altmann et al. (2020b) und Schoenherr et al. (2019b) verwiesen.

\section{Patienten}

Die Stichprobe umfasst 267 Patienten mit diagnostizierter sozialer Angststörung, von denen 111 mithilfe der NATVT, 102 mithilfe der MAN-VT und 54 mithilfe der MAN-TP behandelt wurden. Die Ungleichverteilung der Therapiegruppen war dem Umstand geschul- det, dass für die MAN-TP seltener Videos von erforderlicher Qualität vorlagen. In den manualisierten Therapien war die soziale Angststörung immer die Primärdiagnose, in den naturalistischen Therapien konnte diese die Primär- oder Sekundärdiagnose sein. Es waren 53,6\% der Patienten Frauen. Der Altersdurchschnitt betrug 34,4 Jahre (SD $\pm 11,8$ Jahre). Von den Patienten hatten $59,2 \%$ das Abitur. Die Hälfte $(50,6 \%)$ lebte in einer festen Beziehung (weitere Details: Altmann et al. 2020b).

\section{Behandlung und Therapeuten}

Die MAN-VT (Stangier et al. 2006) basierte auf dem von Clark und Wells (1995) formulierten Modell der sozialen Angststörung. Interventionen waren u. a. die Formulierung eines persönlichen (Krankheits-)Modells, Restrukturierung des Selbstbilds, Übungen zur Reduktion des externen Fokus, rollenspielbasierte Verhaltensexperimente und Videofeedback.

Die MAN-TP (Leichsenring et al. 2007) war eine an die soziale Angststörung angepasste Variante der supportiven-expressiven Therapie nach Luborsky (1984). Zentrale Interventionen waren u.a. die Etablierung einer positiven therapeutischen Beziehung (supportive Interventionen), die Formulierung des zentralen Beziehungskonfliktthemas und dessen Bearbeitung in Bezug auf frühere und aktuelle Beziehungen (expressive Interventionen).

Die NAT-VT folgte einem integrativen Ansatz mit interpersonellen und emotionsfokussierten Elementen (Lutz et al. 2016). Typische Interventionen waren Exposition, kognitive Umstrukturierung, Entspannungstrainings und Psychoedukation. Die Interventionen folgten keinem Manual, sondern orientierten sich am therapeutischen Prozess. Im Gegensatz zu den manualisierten Therapien erhielten die Therapeuten nach jeder Sitzung ein Feedback zum Therapieprozess auf der Basis von PatientenRatings.

Alle Therapien erfolgten im einzeltherapeutischen Setting. Dem deutschen Gesundheitssystem entsprechend waren die ersten 5 Sitzungen immer proba- 
torisch ausgerichtet; in diesen wurden diagnostische und administrative Fragen abgeklärt. Beide manualisierte Therapien waren Kurzzeittherapien (durchschnittliche Zahl der Sitzungen: 26,0 in der VT und 27,1 in der TP). Die NAT-VT erfolgte je nach Indikation und Prozessverlauf im Kurz- oder im Langzeitsetting (im Durchschnitt 42,7 Sitzungen).

In der vorliegenden Studie wurden die Patienten von 119 Therapeuten behandelt $(78 \%$ weiblich, Altersdurchschnitt 31,5 Jahre). Ein Therapeut führte ausschließlich Therapien innerhalb einer Therapiegruppe durch (einer bis 10 Patienten/Therapeut, Median $=2$ Patienten). Alle Therapeuten hatten eine universitäre Ausbildung als Psychologe oder Arzt. Ihre Therapeutenausbildung war abgeschlossen oder sie befanden sich in einem fortgeschrittenen Stadium ihrer Therapeutenausbildung. Bezogen auf die 267 untersuchten Behandlungen wurden $65,9 \%$ von Therapeuten mit einer Therapieerfahrung von 3 oder mehr abgeschlossenen Behandlungen durchgeführt. Die Verteilung erfahrener und weniger erfahrener Therapeuten differierte zwischen den Therapiegruppen (NAT-VT: 80,2\% Therapien mit erfahrenen Therapeuten, MAN-VT: $51,0 \%$, MAN-TP: $64,8 \% ; \chi_{d f=2}^{2}=20,2, p<0,001$, Cramers $V=0,275$ ).

\section{Instrumente}

Als primäres Ergebniskriterium wurde in der vorliegenden Studie die Selbstbeschreibung im Inventar zur Erfassung interpersonaler Probleme (IIP-32; Horowitz et al. 2016) verwendet. Als sekundäres Ergebniskriterium wurde die Selbsteinschätzung im Beck-Depressions-Inventar erfasst (BDI; Beck et al. 1961). Eine naheliegende Anwendung eines störungsspezifischen Maßes (z. B. die Liebowitz-Soziale-Angst-Skala, LSAS) konnte nicht erfolgen, da die angstspezifischen Skalen in den beiden Zentren unterschiedlich waren. Die patientenseitige Beurteilung der therapeutischen Beziehung wurde mithilfe des Helping Alliance Questionnaire (HAQ-I; Bassler et al. 1995; HAQ-II; Luborsky et al. 1996) gemessen. Da in den Zentren verschiedene Versionen des BDI und des HAQ eingesetzt wurden, wurde der Score aus jeweils überlappenden Items betrachtet (BDI: 16 Items, Korrelationen des neuen Scores mit BDI-I und BDIII $r>0,98$; HAQ: 5 Items, Korrelationen mit dem HAQ-I und HAQ-II $r>0,75$ ). Die interne Konsistenz der verwendeten Skalen war gut (Cronbachs $\alpha>0,8$ ). Die Werte in IIP und BDI wurden zu Beginn und am Ende der Therapie gemessen, der HAQ kam nach Sitzung 8 zum Einsatz.

\section{Messung synchroner Bewegungen}

Die Therapievideos wurden mithilfe der Motion Energy Analysis (MEA; Altmann 2013; MATLAB@-Skripte: https:// github.com/10101-00001/MEA) ausgewertet. Um die Bewegungen von Patient und Therapeut $\mathrm{zu}$ separieren, wurden „regions of interest“ (ROI) definiert. Der Algorithmus zählte dann für jeden Videoframe aus, wie viele Pixel der interessierenden ROI ihre Grauintensität im Vergleich zum vorherigen Videoframe substanziell veränderten. Aufdiese Weise wurden pro Video 2 Zeitreihen generiert (eine für den Patienten und eine für den Therapeuten), die den Verlauf der Bewegungsintensität im Millisekundenbereich abbildeten. Um unterschiedliche Körpergrößen bzw. unterschiedlich große ROI auszugleichen, wurden die Motion-Energy-Zeitreihen an der Größe der ROI standardisiert. Der kleinstmögliche Wert war 0 (keine Bewegung bzw. keine Veränderung in der ROI zu diesem Zeitpunkt) und der größtmögliche Wert 100 (extrem raumgreifende Bewegung, sodass zu diesem Zeitpunkt alle Pixel der ROI die Grauintensität veränderten). Der Empfehlung von Schoenherr et al. (2019a) folgend wurden die Zeitreihen im nächsten Schritt mit einem MovingMedian (Bandbreite 5 Frames) geglättet und die Werte log-transformiert.

Für die Berechnung der Synchronisationshäufigkeit wurde anschließend mit jedem Zeitreihenpaar eine "windowed cross-lagged correlation" (WCLC; Altmann 2013; Altmann et al. 2020b; Schoenherr et al. 2019c) durchgeführt. Dieses zeitreihenanalytische Verfahren korreliert Abschnitte der Patienten- und der Therapeutenzeitreihe (sog. Windows), wobei die Position des Referenz-
Window und der Zeitversatz zwischen beiden Windows („time-lag") variiert werden. Der maximal zulässige Timelag betrug $5 \mathrm{~s}$. Die Position des Window wurde immer um einen Frame verschoben („overlapping windows").

Um große Korrelationen überproportional zu gewichten und das Vorzeichen zu eliminieren, wurde jede Kreuzkorrelation zu $\mathrm{R}^{2}$ quadriert. Die $\mathrm{R}^{2}$-Werte wurden in einer Matrix gespeichert, deren Zeile die Position des ReferenzWindow und deren Spalte den Timelag widerspiegelte. Ein „Peak-picking“Algorithmus (PPA; Altmann 2013; Altmann et al. 2020b; Schoenherr et al. 2019c; MATLAB@-Skripte (The MathWorks Inc., Natick, MA, USA): https:// github.com/10101-00001/sync_ident) identifizierte dann auf der Basis der $\mathrm{R}^{2}$ Matrix die Synchronisationsintervalle. Die $\mathrm{R}^{2}$-Matrix wurde grafisch mithilfe eines "surface plot" dargestellt, in dem ein Synchronisationsintervall jeweils mit einem Bergkamm im Plot korrespondiert (Altmann 2013). Der PPA stellte zunächst für jeden Zeitpunkt (bzw. jede Position des Referenz-Window) die Höchstwerte (bzw. im Plot die Peaks) fest. Anschließend wurden zeitlich benachbarte Peaks (Höchstwerte) mit gleichem Time-lag zu einem Bergkamm zusammengefasst. Schließlich wurden die gefundenen Bergkämme gefiltert. Sie mussten eine Mindestgröße von 0,25 aufweisen (der Range für $\mathrm{R}^{2}$ Werte beträgt $0-1$ ). Bei parallel verlaufenden Bergkämmen wurde der höchste (bzw. jener mit den größten Kreuzkorrelationen) ausgewählt. Charakteristika der Bergkämme (Start-, Endzeitpunkt, Time-lag, durchschnittliche $\mathrm{R}^{2}$-Werte) wurden in einer Liste gespeichert („list of sync intervals").

Anhand der Liste wurden verschiedene Kennwerte berechnet: a) wie oft Patient und Therapeut synchronisierten, wobei der Patient der Leader und der Therapeut der Imitierende war (Dauer der Synchronisation mit dem Patienten als Leader in Sekunden), b) wie oft beide synchronisierten und dabei der Therapeut der Leader war (Synchronisation mit dem Therapeuten als Leader), c) wie oft beide synchronisierten (die Summe aus a und b; im Folgenden als „synchrony“ be- 
zeichnet), und d) ob der Patient häufiger als der Therapeut der Leader war (Differenz von a und b; im Folgenden als „leading“ bezeichnet). Um die unterschiedliche Dauer von Videos herauszurechnen, wurde in der vorliegenden Studie die Synchronisationshäufigkeit relativ zur Dauer des jeweiligen Videos betrachtet. Weiterhin ist zu beachten, dass aufgrund der zeitliche dichten MotionEnergy-Messungen während eines Synchronisationsintervalls immer von einem Zeitversatz zwischen dem Verhalten des Drive und des Driven auszugehen ist. Das verwendete Synchrony-Maß bildet insofern etwas ab, das Fraenkel (1983) als „echoing“ bezeichnet.

Da die Computeralgorithmen bei einem Video immer den gleichen Synchronisationswert berechnen (wenn die gleichen Parametereinstellungen verwendet werden), sind Interrater- und Intrarater-Reliabilität als perfekt anzusehen (Altmann 2013). Validierungsstudien (Feniger-Schaal et al. 2020; Schoenherr et al. 2019a, 2019c) konnten die prädiktive, konvergente und kriteriumsbezogene Validität belegen.

In der vorliegenden Studie wurden nur Videos der 8. Therapiesitzung (bzw. der 3. Sitzung der antragspflichtigen Therapie) betrachtet. Falls das Video nicht vorhanden war, wurde das Video der 7. oder 9. Sitzung verwendet. In Anlehnung an Ramseyer und Tschacher (2011) wurden die ersten $15 \mathrm{~min}$ des Videos analysiert, wobei die Begrüßung, das Klären administrativer Fragen und das Ausfüllen von Fragebogen zuvor aus den Videos herausgeschnitten wurden.

\section{Statistische Analysen}

Fehlende Werte auf der Ebene der Summen-Scores der psychometrischen Instrumente und der Synchronisationswerte wurden mit dem R Package missForest (Stekhoven 2011) imputiert. Die folgenden Datenanalysen basieren auf der imputierten „intention-to-treat"-Stichprobe.

Zunächst wurde eine „Grand-mean“Zentrierung der Prädiktoren (Raudenbush und Bryk 2002; Alter des Therapeuten, Pretest [Messung zu Therapiebeginn], therapeutische Beziehung,
Synchrony und Leading) vorgenommen. Dadurch kann der „intercept“ der folgenden Mehrebenenmodelle als der jeweilige Wert für das Therapieergebnis interpretiert werden, der beim „Durchschnittspatienten“ (alle Prädiktoren haben den Durchschnittswert) zu erwarten ist. Im nächsten Schritt erfolgte eine „Group-mean“-Zentrierung (Raudenbush und Bryk 2002) der Prädiktoren. Da mehrere Patienten vom gleichen Therapeuten behandelt wurden, stellt der Mittelwert einer Variablen, gemittelt über alle Patienten eines Therapeuten, den Therapeuten- bzw. Betweentherapist-Anteil dar. Die Abweichung des individuellen Messwerts vom Therapeutenmittelwert repräsentiert den Within-therapist-Anteil. In Anlehnung an Zilcha-Mano (2017) kann der Between-therapist-Anteil als „Trait-like“Komponente des Therapeuten und der Within-therapist-Anteil als "State-like“Komponente interpretiert werden. Allerdings ist $\mathrm{zu}$ beachten, dass es sich dabei um eine stark vereinfachte Interpretation handelt. Genau genommen, müssten für eine solche Interpretation mehrere Allianz- und Synchronisationsmessungen pro Patient vorliegen und ein Patienten durch mindestens 2 Therapeuten behandelt werden, um den Trait des Therapeuten vom Trait des Patienten und von Situationsanteilen separieren zu können.

Für die Untersuchung von Therapeuteneffekten wurden verschiedene Mehrebenenmodelle verwendet. Die abhängigen Variablen waren interpersonale Probleme und Depressionssymptome, gemessen zum Therapieende (IIP post bzw. BDI post). Das Baseline-Modell enthielt die Between- und Within-Variable der jeweiligen Prämessung als Prädiktor (z. B. „IIP pre within“ und „IIP pre between“). Weiterhin wurde ein „random intercept“ mit 2 zufälligen Effekten modelliert: ein Therapeuteneffekt auf Level 2 (mehrere Patienten pro Therapeut) und ein „Treatment“-Effekt auf Level 3 (mehrere Therapeuten pro Therapiegruppe). Aufgrund dieser Parametrisierung bildeten die Regressionskoeffizienten die Zusammenhänge zwischen abhängigen Variablen und unabhängiger Variable im Durchschnitt über alle Therapiegruppen und im Durchschnitt über alle Therapeuten ab. Von einer Differenzierung der Therapiegruppen wurde abgesehen, da in diesem Fall verhältnismäßig viele Interaktionseffekte $\mathrm{zu}$ betrachten wären - bei gleichzeitig relativ kleinen Substichproben (z. B. $n=54$ für MAN-TP). Auch soll darauf hingewiesen sein, dass es nicht das Ziel der vorliegenden Studie war, die Effektivität der 3 Therapien zu vergleichen. Der interessierte Leser wird dahingehend auf die Studien von Leichsenring et al. (2013) und Bögels et al. (2014) verwiesen. Der zufällige Effekt der Therapiegruppe hat die Funktion, zumindest für Effekte des Therapieverfahrens statistisch $\mathrm{zu}$ kontrollieren. Zudem können die ICC für die Therapeuten und Therapiegruppen verglichen werden.

Anschließend wurde das Mehrebenenmodell schrittweise um weitere Prädiktoren erweitert. Die Blöcke waren: 1. Alter und Geschlecht des Therapeuten, 2. Between- und Within-therapistAnteil der therapeutischen Beziehung, 3. Between- und Within-Anteil der Bewegungssynchronisation sowie $\mathrm{Be}$ tween- und Within-therapist-Anteil der Leading-Tendenz in Sitzung 8. Mithilfe des $\chi^{2}$-Tests der Devianzdifferenz des Baseline-Modells und des aktuellen Modells wurde geprüft, ob durch die Hinzunahme eines Blocks von Prädiktoren mehr Varianz der OutcomeVariablen erklärt werden konnte. Der Therapeuteneffekt und der Effekt der Therapiegruppe wurden mit der ICC der Varianzen der entsprechenden zufälligen Effekte quantifiziert. Im letzten Schritt wurde ein Mehrebenenmodell mit allen oben genannten Prädiktoren verwendet.

Das Signifikanzniveau aller Tests war $\alpha=0,05$. Die Mehrebenenanalysen wurden mit den R Packages lme4 Version 1.1.24 (Bates et al. 2015) und lmerTest Version 3.1.1 (Kuznetsova et al. 2017) durchgeführt. Bei jedem Modell wurde der „Restricted-maximumlikelihood“-Schätzer verwendet.

\section{Ergebnisse}

Die Statistiken zur Vorhersage der interpersonalen Probleme am Therapieende können $\bullet$ Tab. 1 entnommen werden. Die 
Tab. 1 Mehrebenenmodelle zur Vorhersage der Patienten-Ratings der interpersonellen Probleme am Therapieende (IIP post)

\begin{tabular}{|c|c|c|c|c|c|c|c|c|c|c|c|c|c|c|c|c|c|c|}
\hline \multirow[b]{2}{*}{ Prädiktoren } & \multicolumn{3}{|c|}{$\begin{array}{l}\text { Modell } 0 \\
\text { (M0) }\end{array}$} & \multicolumn{3}{|c|}{$\begin{array}{l}\text { Modell } 1 \\
\text { (M1) }\end{array}$} & \multicolumn{3}{|c|}{$\begin{array}{l}\text { Modell } 2 \\
\text { (M2) }\end{array}$} & \multicolumn{3}{|c|}{$\begin{array}{l}\text { Modell } 3 \\
\text { (M3) }\end{array}$} & \multicolumn{3}{|c|}{$\begin{array}{l}\text { Modell } 4 \\
\text { (M4) }\end{array}$} & \multicolumn{3}{|c|}{$\begin{array}{l}\text { Modell } 5 \\
\text { (M5) }\end{array}$} \\
\hline & $b$ & $S E$ & $\beta$ & $b$ & $S E$ & $\beta$ & $b$ & $S E$ & $\beta$ & $b$ & $S E$ & $\beta$ & $b$ & $S E$ & $\beta$ & $b$ & $S E$ & $\beta$ \\
\hline Intercept & $1,34^{* * *}$ & 0,10 & $-0,10$ & $1,38^{* * *}$ & 0,12 & $-0,03$ & $1,41^{* * *}$ & 0,12 & $-0,05$ & $1,37^{* * *}$ & 0,11 & $-0,05$ & $1,36^{* * *}$ & 0,07 & $-0,07$ & $1,35^{* * *}$ & 0,08 & $-0,11$ \\
\hline BDI pre within & - & - & - & $0,58^{* * * *}$ & 0,07 & 0,33 & $0,58^{* * *}$ & 0,07 & 0,34 & $0,58^{* * *}$ & 0,07 & 0,33 & $0,60^{* * * *}$ & 0,07 & 0,35 & $0,61^{* * * *}$ & 0,07 & 0,35 \\
\hline $\begin{array}{l}\text { BDI pre be- } \\
\text { tween }\end{array}$ & - & - & - & $0,65^{* * *}$ & 0,11 & 0,39 & $0,64^{* * *}$ & 0,12 & 0,38 & $0,63^{* * *}$ & 0,11 & 0,38 & $0,67^{* * *}$ & 0,11 & 0,40 & $0,64^{* * *}$ & 0,11 & 0,38 \\
\hline $\begin{array}{l}\text { Therapeuten- } \\
\text { alter (Jahre) }\end{array}$ & - & - & - & - & - & - & 0,01 & 0,01 & 0,08 & - & - & - & - & - & - & 0,00 & 0,01 & 0,07 \\
\hline $\begin{array}{l}\text { Therapeuten- } \\
\text { geschlecht } \\
\text { (1 = weiblich) }\end{array}$ & - & - & - & - & - & - & $-0,05$ & 0,08 & $-0,04$ & - & - & - & - & - & - & $-0,02$ & 0,09 & $-0,02$ \\
\hline HAQ S8 within & - & - & - & - & - & - & - & - & - & 0,00 & 0,07 & 0,00 & - & - & - & 0,02 & 0,07 & 0,02 \\
\hline $\begin{array}{l}\text { HAQ S8 be- } \\
\text { tween }\end{array}$ & - & - & - & - & - & - & - & - & - & $-0,20^{*}$ & 0,10 & $-0,14$ & - & - & - & $-0,19$ & 0,10 & $-0,13$ \\
\hline $\begin{array}{l}\text { Synchrony S8 } \\
\text { within }\end{array}$ & - & - & - & - & - & - & - & - & - & - & - & - & $-0,01^{*}$ & 0,00 & $-0,11$ & $-0,01^{*}$ & 0,00 & $-0,12$ \\
\hline $\begin{array}{l}\text { Synchrony S8 } \\
\text { between }\end{array}$ & - & - & - & - & - & - & - & - & - & - & - & - & $-0,02^{* *}$ & 0,01 & $-0,23$ & $-0,02^{* *}$ & 0,01 & $-0,23$ \\
\hline $\begin{array}{l}\text { Leading S8 } \\
\text { within }\end{array}$ & - & - & - & - & - & - & - & - & - & - & - & - & 0,02 & 0,01 & 0,06 & 0,02 & 0,01 & 0,07 \\
\hline $\begin{array}{l}\text { Leading S8 } \\
\text { between }\end{array}$ & - & - & - & - & - & - & - & - & - & - & - & - & 0,03 & 0,02 & 0,10 & 0,03 & 0,02 & 0,09 \\
\hline \multicolumn{19}{|l|}{ Random Effects } \\
\hline Var(e) (Dyade) & \multicolumn{3}{|l|}{0,16} & \multicolumn{3}{|l|}{0,12} & \multicolumn{3}{|l|}{0,12} & \multicolumn{3}{|l|}{0,11} & \multicolumn{3}{|l|}{0,11} & \multicolumn{3}{|l|}{0,11} \\
\hline $\begin{array}{l}\operatorname{Var}\left(\mathrm{u}_{0}\right) \text { (Thera- } \\
\text { peut) }\end{array}$ & \multicolumn{3}{|l|}{0,08} & \multicolumn{3}{|l|}{0,06} & \multicolumn{3}{|l|}{0,06} & \multicolumn{3}{|l|}{0,06} & \multicolumn{3}{|l|}{0,05} & \multicolumn{3}{|l|}{0,06} \\
\hline $\begin{array}{l}\operatorname{Var}\left(\mathrm{u}_{00}\right) \text { (The- } \\
\text { rapiearm) }\end{array}$ & \multicolumn{3}{|l|}{0,03} & \multicolumn{3}{|l|}{0,04} & \multicolumn{3}{|l|}{0,03} & \multicolumn{3}{|l|}{0,03} & \multicolumn{3}{|l|}{0,01} & \multicolumn{3}{|l|}{0,00} \\
\hline $\begin{array}{l}\text { Marginal } R^{2} / \\
\text { Conditional } R^{2}\end{array}$ & \multicolumn{3}{|c|}{$0,000 / 0,408$} & \multirow{2}{*}{\multicolumn{3}{|c|}{$0,243 / 0,601$}} & \multicolumn{3}{|c|}{$0,260 / 0,587$} & \multicolumn{3}{|c|}{$0,265 / 0,593$} & $0,331 / 0$ & 0,573 & & $0,376 / 0$ & 0,588 & \\
\hline Deviance & 453,25 & & & & & & 372,305 & & & 369,722 & & & $355,81 \varepsilon$ & & & 348,915 & & \\
\hline
\end{tabular}

soziodemografischen Variablen (Modell M2) und die Leading-Tendenz (M4) waren nicht für dieses Outcome prädiktiv. Hingegen waren Within-therapist-Anteil von IIP pre (M1: $b=0,58$, $p<0,001)$, Between-therapist-Anteil von IIP pre (M1: $b=0,65, p<0,001), B e-$ tween-Anteil der Allianz (M3: $b=-0,20$, $p<0,05)$, Between-Anteil der Synchronisation (M4: $b=-0,02, p<0,01)$ und Within-Anteil der Synchronisation (M4: $b=-0,01, p<0,01)$ signifikant mit dem Outcome IIP post assoziiert. Werden alle Prädiktoren in das Modell eingeschlossen, erreichten nur noch die beiden Synchronisationsvariablen (M5: within $b=-0,01, p<0,01$, between $b=-0,02$, $p<0,01)$ das erforderliche Signifikanzniveau. Ein Vergleich der "model fits“ zeigte, dass M4 den besten Fit hatte. Zwar wies M5 die geringste Devianz auf, jedoch war die Reduktion der Devianz von M4 zu M5 nicht signifikant $\left(\chi^{2}=6,9\right.$, $\mathrm{df}=4, p=0,1396)$. Die durch die zufälligen Therapeuteneffekte erklärte Varianz am Therapieergebnis (ICC $=0,301$ ) war deutlich größer als jene der TreatmentGruppe (ICC =0,060). Beim Vergleich von M1 und M4 hinsichtlich der ICC der zufälligen Therapeuten- und Behandlungseffekte zeigte sich, dass die Hinzunahme der Synchronisationsva- riablen die Varianz des zufälligen Effekts für die Therapiegruppe reduzierte (M1: $\operatorname{Var}\left[\mathrm{u}_{00}\right]=0,04$ vs. M4: $\left.\operatorname{Var}\left[\mathrm{u}_{00}\right]=0,01\right)$.

Das gleiche Prozedere wurde auch bei den Depressionssymptomen am Therapieende, gemessen mit dem BDI, angewendet (•Tab. 2). Hier waren das Alter des Therapeuten (M2: $b=-1,71, p<0,05)$, der Between-therapist-Anteil der Allianz (M3: $b=-3,34, p<0,001)$ und der Between-therapist-Anteil der LeadingTendenz (M4: $b=0,50, p<0,05)$ signifikant mit dem Outcome BDI post assoziiert. Im Modell mit allen Prädiktoren (M5) waren nur BDI-pre-Within-therapist-Anteil $(b=0,31, p<0,001)$, BDI- 


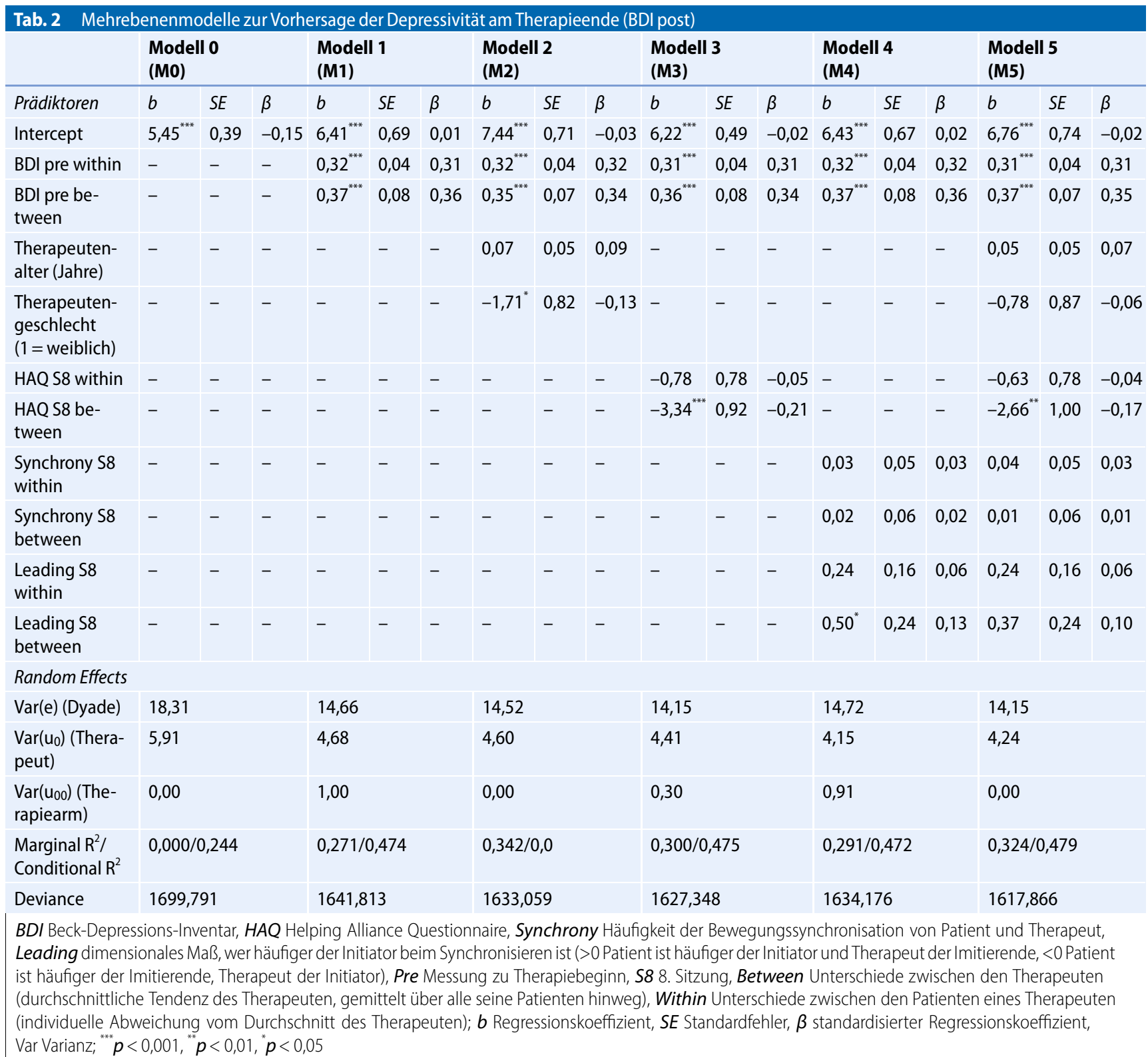

pre-Between-Therapist-Anteil $(b=0,37$, $p<0,001)$ und HAQ-Between-Anteil $(b=-2,66, p<0,01)$ für die Vorhersage bedeutsam. Modellvergleiche legen die Präferenz von M3 nahe. Zwar hatte M5 die geringste Devianz, aber durch die Hinzunahme weiterer Prädiktoren konnte der Fit von M3 zu M5 nicht signifikant verbessert werden $\left(\chi^{2}=9,2, \mathrm{df}=6\right.$, $p=0,1722)$. Auch bei den Depressionssymptomen erklärten die zufälligen Therapeuteneffekte (M3: ICC $=0,234)$ mehr Varianz als der zufällige Effekt der Behandlungsgruppe (ICC $=0,016)$. Ebenfalls reduzierte sich maßgeblich die Varianz des zufälligen Effekts für die
Therapiegruppe durch die Hinzunahme der therapeutischen Beziehung als Prädiktor (M1: $\operatorname{Var}\left[\tau_{00}\right]=1,00$ vs. M3: $\left.\operatorname{Var}\left[\tau_{00}\right]=0,30\right)$ und nicht die Varianz der zufälligen Therapeuteneffekte.

\section{Diskussion}

\section{Interpretation der Ergebnisse und Vergleich mit der Literatur}

Die vorliegende Studie untersuchte mit 3 methodischen Ansätzen Therapeuteneffekte bei der ambulanten Behandlung der sozialen Angststörung. Zum einen wurde mit Mehrebenenmodellen und zufälligen
Effekten untersucht, welcher Anteil der Outcome-Varianz durch die Therapeutenvariable erklärt werden kann. Im Vergleich zu den Durchschnittswerten der Metaanalysen von Crits-Christoph et al. (1991), Baldwin und Imel (2013) und Johns et al. (2019; ICC = 5-8,6\%) sind die ermittelten ICC für interpersonelle Probleme (ICC $=30,1 \%$ in M4) und Depressionssymptome (ICC $=23,4 \%$ in M3) als sehrgroß einzuordnen. Auch sind bei den vorliegenden Analysen die Therapeuteneffekte deutlich größer als die ICC für die Therapiegruppen (ICC $=6,0 \%$ bzw. ICC $=1,6 \%)$. Die Befunde legen nahe, dass in der vorliegenden Stichprobe von 
Patienten mit sozialer Angststörung die Person des Therapeuten für die Verbesserung der interpersonalen Probleme und der Depressivität bedeutsamer war als das angewendete Therapieverfahren, und dass Therapeuteneffekte je nach Outcome unterschiedlich ausgeprägt sind.

Der zweite methodische Ansatz betrachtete spezifische Therapeutenvariablen. Alter und Geschlecht des Therapeuten waren wie in anderen Studien (Bowman et al. 2001; Chow et al. 2015; Huppert et al. 2001) nicht für den Therapieerfolg prädiktiv (M4 in • Tab. 1 und M3 in - Tab. 2). In Anlehnung an Bowman et al. (2001) kann geschlussfolgert werden, dass Alter und Geschlecht des Therapeuten kaum Relevanz für die Planung von Psychotherapien sozialer Angststörungen haben sollten.

Der dritte methodische Ansatz disaggregierte dyadische prozessrelevante Variablen in einen Therapeuten- und Patientenanteil bzw. in einen Betweentherapist- und einen Within-therapistAnteil. Insgesamt zeigen die Befunde, dass sich bei Messungen der therapeutischen Beziehung und der nonverbalen Synchronisation Therapeutenanteile separieren lassen und dass diese Betweentherapist-Anteile für den Therapieerfolg weit aus relevanter sind als die Within-therapist-Anteile. Der Betweentherapist-Anteil ist der Durchschnitt der therapeutischen Allianz (bzw. der Bewegungssynchronisation) über alle Dyaden mit diesen Therapeuten hinweg. Klinisch kann dies als Fähigkeit des Therapeuten/der Therapeutin interpretiert werden, wie gut er oder sie es schafft, eine therapeutische Beziehung aufzubauen (eine „Trait-like“-Komponente). Der Between-therapist-Anteil gibt somit auch an, wie gut der Therapeut im Vergleich zu anderen Therapeuten ist. Der Within-therapist-Anteil ist die Abweichung des individuellen Messwerts vom Therapeutendurchschnitt und beschreibt die Unterschiede bezüglich der Messungen von Patienten eines spezifischen Therapeuten. In Übereinstimmung mit anderen Studien (Baldwin et al. 2007; Hara et al. 2017; Zuroff et al. 2010) sagte der Between-therapist-Anteil der therapeutischen Beziehung die Verbesserung der Depressivität vorher (Modell M3 in
- Tab. 2). Mit anderen Worten: Für Verbesserungen im affektiven Bereich war die allgemeine Fähigkeit eines Therapeuten zum Beziehungsaufbau in der ersten Therapiephase (Between-therapist-Anteil) relevant. Weniger bedeutsam war hingegen die individuelle Beziehung zum Patienten in Relation zur generellen Fähigkeit zum Beziehungsaufbau des Therapeuten (Within-therapist-Anteil).

Die Häufigkeit der Bewegungssynchronisation (Movement synchrony) von Patient und Therapeut während der Therapiesitzung - die zweite der betrachteten prozessrelevanten Variablen - war ebenfalls prädiktiv für den Therapieerfolg. Vergleichbar mit der Studie von Ramseyer und Tschacher (2011) sagte eine häufige Synchronisation die Reduktion interpersoneller Probleme vorher. Interessanterweise waren hier sowohl Between- als auch Within-therapist-Anteile prädiktiv. Die Betweentherapist-Anteile wiesen jedoch die betragsmäßig größeren standardisierten Regressionskoeffizienten auf (M4 in - Tab. 1: $\beta=-0,11$ vs. $\beta=-0,23)$. Dies legt nahe, dass die allgemeine Fähigkeit des Therapeuten, mit Patienten auf der Bewegungsebene zu synchronisieren (der Between-therapist-Anteil), um z. B. Zustimmung (Keller und Tschacher 2007) oder Empathie (Fraenkel 1983) zu signalisieren, für Verbesserungen interpersoneller Probleme bedeutsam ist. Ihr kommt mehr Gewicht zu als der in der Dyade gezeigten Bewegungssynchronisation in Relation zum Durchschnitt der Bewegungssynchronisation in Dyaden mit diesem Therapeuten (Withintherapist-Anteil).

Weiterhin zeigten Modelle, in denen therapeutische Beziehung und Synchronisation gleichzeitig als Prädiktoren verwendet wurden (jeweils Modell M5 in - Tab. 1 und 2), dass die jeweiligen Between-therapist-Anteile für unterschiedliche Bereiche relevant sind und sich gegenseitig ausschließen: Häufige Synchronisation korrelierte mit einer Reduktion interpersoneller Probleme. Die therapeutische Beziehung war bei diesem Outcome jedoch nicht prädiktiv. Dies legt nahe, dass die Fähigkeit der Therapeuten, nonverbal synchron zu kommunizieren, für die Therapie interpersonel- ler Probleme und die damit verbundene Bewältigung maladaptiver Interaktionsmuster von größerer Relevanz ist als die patientenseitige Wahrnehmung der therapeutischen Allianz. Im Gegensatz dazu war die nonverbale Synchronisation für Verbesserungen im affektiven Bereich nicht prädiktiv. Vielmehr folgte auf eine als sehr gut eingeschätzte therapeutische Beziehung eine Reduktion der Depressivität. Offenbar sind hier therapeutische Techniken zur Stärkung der therapeutischen Beziehung wie Exploration, Reflexion, Vergegenwärtigung vergangener Erfolge der Therapie, genaue Interpretation, Erleichterung des Ausdrucks des Affekts und die Berücksichtigung der Erfahrungen des Patienten (Ackerman und Hilsenroth 2003) wichtiger als die nonverbale Kommunikation.

Bezüglich des Verhältnisses von therapeutischer Beziehung und nonverbaler Synchronisation ist auch die von Wiltshire et al. (2020) aufgeworfene Frage in Erinnerung zu rufen, ob therapeutische Beziehung und Synchronisation Facetten eines übergeordneten Konstrukts sind oder Synchronisation eher ein eigenständiges therapierelevantes Konstrukt ist. Die Befunde der vorliegenden Studie legen eher Letzteres nahe. Dies könnte erklären, warum Studien zur Assoziation von Synchronisation und Allianz keine hohen Korrelationen finden, sondern allenfalls moderate (z.B. $r=0,33$ bei Ramseyer und Tschacher 2011) bis hin zu nichtsignifikanten Zusammenhängen (Paulick et al. 2018). Zukünftige Therapieforschung sollte deshalb Therapeuteneffekte stärker in den Blick nehmen. Insbesondere erhebt sich die Frage, welchen Einfluss Therapeutencharakteristika wie z.B. Empathiefähigkeit auf die nonverbale Kommunikation, den Therapieprozess und das Therapieergebnis bei sozialen Ängsten haben.

Und schließlich soll darauf hingewiesen sein, dass sich die Zusammenhänge zwischen nonverbaler PatientTherapeut-Kommunikation und Therapieergebnis ausschließlich auf Körperbewegungen und deren Synchronisation beziehen. Erst kürzlich wurde deutlich gemacht, dass körperlicher Aktivität in der Prävention und Behandlung von Angsterkrankungen eine weitaus größe- 
re Bedeutung beizumessen ist, als dies bisher der Fall ist (Petzold et al. 2020).

\section{Limitation der Studie}

Zu den Limitationen der Studie zählt, dass keine Veränderungen sozialer Ängste betrachtet wurden. Die Hauptstudie nutzte Videodatenbanken aus 2 Zentren, die, wie erwähnt, unterschiedliche Instrumente zur Messung sozialer Ängste einsetzten, sodass kein zentrenübergreifendes Angstinstrument vorlag.

Eine weitere wesentliche Limitation ist die Stichprobengröße mit 267 Patienten, die im Vergleich zu den für Mehrebenenanalysen mit zufälligen Effekten empfohlenen Stichprobengrößen als klein zu bezeichnen ist (Schiefele et al. 2017). Zudem wird das für Mehrebenenanalysen empfohlene Verhältnis von 30 Patienten/ Therapeut (Kreft und De Leeuw 1998) mit durchschnittlich 2 Patienten/Therapeut deutlich unterschritten. Allerdings zeigten spätere Simulationsstudien, dass eine große Zahl von Level-2-Units (hier die Therapeuten) von größerer Relevanz ist (Maas und Hox 2005) - was in der vorliegenden Studie wiederum gegeben ist. Ziel der Hauptstudie war es allerdings, Zusammenhänge zwischen nonverbaler Synchronisation und Therapieerfolg zu untersuchen. Therapeuteneffekte standen bei der Planung der Hauptstudie nicht im Fokus. Im Vergleich zu anderen Synchronisationsstudien ist die vorliegende Stichprobe als groß einzuschätzen (z. B. $n=93$ bei Paulick et al. 2018; $n=70$ bei Ramseyer und Tschacher 2011). Gemäß Schiefele et al. (2017, S. 609) ist bei der vorliegenden Datensituation eine Überschätzung der Varianzen der zufälligen Therapeuteneffekte zu erwarten. Die Verallgemeinerbarkeit der Ergebnisse zu den ICC sowie den Between- und Within-therapist-Effekten ist deshalb eingeschränkt.

Eine Limitation der Studie betrifft das Design. Für eine Entflechtung von Effekten des Therapeuten, des Patienten, der spezifischen Dyade und von Situationseffekten wäre es nach Baldwin und Imel (2013) notwendig, dass (1.) pro Dyade mehrere Messungen z.B. der therapeutischen Beziehung vorliegen, (2.) Therapeuten mehrere Patienten behandeln und
(3.) Patienten im Laufe ihrer Therapie von mindestens 2 Therapeuten behandelt werden („round robin design“). Die vorliegende Studie erfüllt nur Punkt (2.), sodass Patienten- und Dyadenanteile nicht klar trennbar sind. Die Berücksichtigung der 3 Punkte bedürfte allerdings einer schwierigen und aufwendigen Planung.

\section{Fazit für die Praxis}

- Die vorliegende Studie illustrierte drei methodische Ansätze zur Untersuchung von Therapeuteneffekten. Insgesamt legt die Studie nahe, dass Therapeuteneffekte bei der Behandlung der sozialen Angststörung von großer Bedeutung sind. Allerdings gilt es zu differenzieren.

- Während soziodemografische Therapeutenvariablen erwartungsgemäß als wenig ergebnisrelevant einzuordnen sind, belegen die Analysen unspezifischer Therapeuteneffekte (Varianzen zufälliger Effekte), dass diese einen stärkeren Einfluss auf das Therapieergebnis bei der Behandlung sozialer Ängste hatten als das Therapieverfahren.

- Darüber hinaus war für den affektiven Bereich (genauer: für depressive Symptome) v.a. das Vermögen der Therapeuten relevant, eine gute therapeutische Beziehung in der ersten Therapiephase aufzubauen.

- Für die Reduktion interpersonaler Probleme war hingegen die allgemeine Fähigkeit des Therapeuten bedeutsam, in der frühen Therapiephase öfter mit ihren Patienten nonverbal zu synchronisieren.

\section{Korrespondenzadresse}

\section{Dr. phil. Uwe Altmann}

Institut für Psychosoziale Medizin,

Psychotherapie und Psychoonkologie,

Universitätsklinikum Jena

Stoystr. 3, 07740 Jena, Deutschland

uwe.altmann@med.uni-jena.de

Danksagung. Ein Teil der in dieser Arbeit beschriebenen Stichprobe entstammt einem Teilprojekt (B1) des Verbundprojekts SOPHO-NET, das durch das Bundesministerium für Bildung und Forschung (BMBF) gefördert wurde (FKZ 01GV0607). Das hier beschriebene Vorhaben wurde von der Deutschen Forschungsgemeinschaft (DFG) in dem Projekt "Ti- ming of nonverbal patient-therapist-interaction and therapeutic success of social phobic patients (TIMPATHIN)" gefördert (FKZ STR 306/28-1 und LU 660/81).

Funding. Open Access funding provided by Projekt DEAL.

\section{Einhaltung ethischer Richtlinien}

Interessenkonflikt. U. Altmann, B. Schwartz, D. Schönherr, J. Rubel, U. Stangier, W. Lutz und B. Strauß geben an, dass kein Interessenkonflikt besteht.

Die Autoren erklären, dass die Forschung ethisch korrekt in Übereinstimmung mit der Deklaration von Helsinki durchgeführt wurde. Der Ethikantrag wurde an die Ethikkommission des Universitätsklinikums Jena, Jena, Deutschland, gestellt (ID 5043-01/17) und von ihr bewilligt. Die Teilnahme an der Studie war freiwillig. Von jedem Teilnehmer wurde eine schriftliche Einwilligungserklärung eingeholt.

Open Access. Dieser Artikel wird unter der Creative Commons Namensnennung 4.0 International Lizenz veröffentlicht, welche die Nutzung, Vervielfältigung, Bearbeitung, Verbreitung und Wiedergabe in jeglichem Medium und Format erlaubt, sofern Sie den/die ursprünglichen Autor(en) und die Quelle ordnungsgemäß nennen, einen Link zur Creative Commons Lizenz beifügen und angeben, ob Änderungen vorgenommen wurden.

Die in diesem Artikel enthaltenen Bilder und sonstiges Drittmaterial unterliegen ebenfalls der genannten Creative Commons Lizenz, sofern sich aus der Abbildungslegende nichts anderes ergibt. Sofern das betreffende Material nicht unter der genannten Creative Commons Lizenz steht und die betreffende Handlung nicht nach gesetzlichen Vorschriften erlaubt ist, ist für die oben aufgeführten Weiterverwendungen des Materials die Einwilligung des jeweiligen Rechteinhabers einzuholen.

Weitere Details zur Lizenz entnehmen Sie bitte der Lizenzinformation auf http://creativecommons.org/ licenses/by/4.0/deed.de.

\section{Literatur}

Ackerman SJ, Hilsenroth MJ (2003) A review of therapist characteristics and techniques positively impacting the therapeutic alliance. Clin Psychol Rev 23(1):1-33. https://doi.org/10.1016/S02727358(02)00146-0

Almlöv J, Carlbring P, Källqvist K, Paxling B, Cuijpers P, Andersson $G$ (2010) Therapist effects in guided Internet-delivered CBT for anxiety disorders. Behav Cogn Psychother 39(3):311-322. https:// doi.org/10.1017/S135246581000069X

Altmann U (2013) Synchronisation nonverbalen Verhaltens. Springer, Berlin (Synchronization of nonverbal behavior)

Altmann U, Gawlytta R, Hoyer J, Leichsenring F, Leibing E, Beutel Metal (2020a) Typical symptom change patterns and their predictors in patients with social anxiety disorder: a latent class analysis. J Anxiety Disord 71:102200. https://doi. org/10.1016/j.janxdis.2020.102200 
Altmann U, Schoenherr D, Paulick J, Deisenhofer A-K, Schwartz B, Rubel JA et al (2020b) Associations between movement synchrony and outcome in patients with social anxiety disorder: evidence for treatment specific effects. Psychother Res 30(5):574-590. https://doi.org/10.1080/ 10503307.2019.1630779

Andersson G, Paxling B, Wiwe M, Vernmark K, Felix CB, Lundborg $L$ et al (2012) Therapeutic alliance in guided internet-delivered cognitive behavioural treatment of depression, generalized anxiety disorder and social anxiety disorder. Behav Res Ther 50(9):544-550. https://doi.org/10.1016/j. brat.2012.05.003

Baldwin SA, Imel ZE (2013) Therapist effects: findings and methods. In: Lambert MJ (Hrsg) Bergin and Garfield's handbook of psychotherapy and behavior change. Wiley, New York, S258-297

Baldwin SA, Wampold BE, Imel ZE (2007) Untangling the alliance-outcome correlation: exploring the relative importance of therapist and patient variability in the alliance. J Consult Clin Psychol 75(6):842-852. https://doi.org/10.1037/0022006X.75.6.842

Barkowski S, Schwartze D, Strauss B, Burlingame GM, Barth J, Rosendahl J (2016) Efficacy of group psychotherapy for social anxiety disorder: a meta-analysis of randomized-controlled trials. J Anxiety Disord 39:44-64. https://doi.org/10. 1016/j.janxdis.2016.02.005

Bassler M, Potratz B, Krauthauser H (1995) Der "Helping Alliance Questionnaire" (HAQ) von Luborsky. Möglichkeiten zur Evaluation des therapeutischen Prozesses von stationärer Psychotherapie. Psychotherapeut 40(1):23-32

Bates D, Mächler M, Bolker B, Walker S (2015) Fitting linear mixed-effects models using \{lme4\}. J Stat Soft 67(1):1-48. https://doi.org/10.18637/jss. v067.i01

Beck AT, Ward CH, Mendelson M, Mock J, Erbaugh J (1961) An inventory for measuring depression. Arch Gen Psychiatry 4(6):561-571. https://doi. org/10.1001/archpsyc.1961.01710120031004

Bögels SM, Wijts P, Oort FJ, Sallaerts SJ (2014) Psychodynamic psychotherapy versus cognitive behavior therapy for social anxiety disorder: an efficacy and partial effectiveness trial. Depress Anxiety31(5):363-373.https://doi.org/10.1002/ da.22246

Bohn C, Aderka IM, Schreiber F, Stangier U, Hofmann SG (2013) Sudden gains in cognitive therapy and interpersonal therapy for social anxiety disorder. JConsult Clin Psychol 81(1):177-182. https://doi. org/10.1037/a0031198

Bowman D, Scogin F, Floyd M, McKendree-Smith N (2001) Psychotherapy length of stay and outcome: a meta-analysis of the effect of therapist sex. Psychother Theory Res Pract Train 38(2):142. https://doi.org/10.1037/0033-3204. 38.2.142

Chow DL, Miller SD, Seidel JA, Kane RT, Thornton JA, Andrews WP (2015) The role of deliberate practice in the development of highly effective psychotherapists. Psychotherapy 52(3):337-345. https:// doi.org/10.1037/pst0000015

Clark DM, Wells A (1995) A cognitive model of social phobia. In: Heimberg RG, Liebowitz MR, Hope DA, Schneier FR (Hrsg) Social phobia: diagnosis, assessment, and treatment. Guilford, New York, NY, S69-93

Constantino MJ, Boswell JF, Coyne AE, Kraus DR, Castonguay LG (2017) Who works for whom and why? Integrating therapist effects analysis into psychotherapy outcome and process research.
In: Castonguay LG, Hill CE (Hrsg) How and why are some therapists better than others? Understanding therapist effects. American Psychological Association, Washington DC, S55-68

Crits-Christoph P, Baranackie K, Kurcias J, Beck A, Carroll K, Perry K et al (1991) Meta-analysis of therapist effects in psychotherapy outcome studies. Psychother Res 2(1):81-91. https://doi org/10.1080/10503309112331335511

Del Re AC, Flückiger C, Horvath AO, Symonds D, Wampold BE (2012) Therapist effects in the therapeutic alliance-outcome relationship: a restricted-maximum likelihood meta-analysis. Clin Psychol Rev 32(7):642-649. https://doi.org/ 10.1016/j.cpr.2012.07.002

Delgadillo J, Branson A, Kellett S, Myles-Hooton P, Hardy GE, Shafran R (2020) Therapist personality traits as predictors of psychological treatment outcomes. Psychother Res. https://doi.org/10. 1080/10503307.2020.1731927

Feldman R (2012) Parent-infant synchrony: a biobehavioral model of mutual influences in the formation of affiliative bonds. Monogr Soc Res Child Dev 77(2):42-51. https://doi.org/10.1111/ j.1540-5834.2011.00660.x

Feniger-Schaal R, Schoenherr D, Altmann U, Strauss B (2020) Movement synchrony in the Mirror Game. JNonverbal Behav. (accepted)

Flückiger C, Del Re A, Wampold BE, Symonds D, Horvath AO (2012) How central is the alliance in psychotherapy? A multilevel longitudinal metaanalysis. J Couns Psychol 59(1):10. https://doi. org/10.1037/a0025749

Flückiger C, Del Re A, Wampold BE, Horvath AO (2018) The alliance in adult psychotherapy: a meta-analytic synthesis. Psychotherapy 55(4):316-340. https://doi.org/10.1037/pst0000172

Fraenkel DL (1983) The relationship of empathy in movement to synchrony, echoing, and empathy in verbal interactions. Am J Dance Ther 6(1):31-48

Hara KM, Aviram A, Constantino MJ, Westra HA, Antony MM (2017) Therapist empathy, homework compliance, and outcome in cognitive behavioral therapy for generalized anxiety disorder: partitioning within- and between-therapist effects. Cogn Behav Ther 46(5):375-390. https:// doi.org/10.1080/16506073.2016.1253605

Haug T, Nordgreen T, Öst L-G, Tangen T, Kvale G, Hovland OJ et al (2016) Working alliance and competence as predictors of outcome in cognitive behavioral therapy for social anxiety and panic disorder in adults. Behav Res Ther 77:40-51. https://doi.org/10.1016/j.brat.2015. 12.004

Heinonen E, Nissen-Lie HA (2020) The professional and personal characteristics of effective psychotherapists: a systematic review. Psychother Res 30(4):417-432. https://doi.org/10.1080/ 10503307.2019.1620366

Herbert JD, Gaudiano BA, Rheingold AA, Moitra E, Myers VH, Dalrymple KL et al (2009) Cognitive behavior therapy for generalized social anxiety disorderin adolescents: a randomized controlled trial. J Anxiety Disord 23(2):167-177. https://doi. org/10.1016/j.janxdis.2008.06.004

Horowitz LM, Strauß B, Thomas A, Kordy H (2016) InventarzurErfassung interpersonalerProbleme - deutsche Version, 3. Aufl. Hogrefe, Göttingen

Horvath AO, Del Re A, Flückiger C, Symonds D (2011) Alliance in individual psychotherapy. Psychotherapy 48(1):9. https://doi.org/10.1037/ a0022186
Hunger C, Hilzinger R, Bergmann NL, Mander J, Bents H, Ditzen B et al (2018) Bezugspersonenbelastung erwachsener Patienten mit sozialer Angststörung. Psychotherapeut 63(3):204-212. https:// doi.org/10.1007/s00278-018-0281-5

Huppert JD, Bufka LF, Barlow DH, Gorman JM, Shear MK, Woods SW (2001) Therapists, therapist variables, and cognitive-behavioral therapy outcome in a multicenter trial for panic disorder. J Consult Clin Psychol 69(5):747-755. https://doi.org/10. 1037/0022-006X.69.5.747

Johns RG, Barkham M, Kellett S, Saxon D (2019) A systematic review of therapist effects: a critical narrative update and refinement to Baldwin and Imel's (2013) review. Clin Psychol Rev 67:78-93. https://doi.org/10.1016/j.cpr.2018.08.004

Keller E, Tschacher W (2007) Prosodic and gestural expression of interactional agreement. In: Esposito A, Faundez-Zanuy M, Keller E, Marinaro M (Hrsg) Verbal and nonverbal communication behaviours. Springer, Berlin, S85-98

Kessler RC, Petukhova M, Sampson NA, Zaslavsky AM, Wittchen HU (2012) Twelve-month and lifetime prevalence and lifetime morbid risk of anxiety and mood disorders in the United States. Int J Methods Psychiatr Res 21(3):169-184. https:// doi.org/10.1002/mpr.1359

Koole SL, Tschacher W (2016) Synchrony in psychotherapy: a review and an integrative framework for the therapeutic alliance. Front Psychol. https:// doi.org/10.3389/fpsyg.2016.00862

Kreft IGG, De Leeuw J (1998) Introducing multilevel modeling. SAGE, Newbury Park, CA

Kuznetsova A, Brockhoff PB, Christensen RHB (2017) \{lmerTest\} package: tests in linear mixed effects models. J Stat Soft 82(13):1-26. https://doi.org/ 10.18637/jss.v082.i13

Leichsenring F, Salzer S, Beutel ME, Herpertz S, Hiller W, Hoyer J et al (2013) Psychodynamic therapy and cognitive-behavioral therapy in social anxiety disorder: a multicenter randomized controlled trial. Am J Psychiatry 170(7):759-767. https:// doi.org/10.1176/appi.ajp.2013.12081125

Leichsenring F, Salzer S, Beutel ME, Herpertz S, Hiller W, Hoyer J et al (2014) Long-term outcome of psychodynamic therapy and cognitivebehavioral therapy in social anxiety disorder. Am JPsychiatry 171(10):1074-1082. https://doi.org/ 10.1176/appi.ajp.2014.13111514

Leichsenring F, Beutel M, Leibing E (2007) Psychodynamic psychotherapy for social phobia: a treatment manual based on supportive-expressive therapy. Bull Menninger Clin 71(1):56-84. https:// doi.org/10.1521/bumc.2007.71.1.56

Luborsky L (1984) Principles of psychoanalytic psychotherapy: a manual for supportiveexpressive (SE) treatment. Basic Books, New York, NY

Luborsky L, Barber JM, Siqueland L, Johnson S, Najavits LM, Frank A et al (1996) The revised helping alliance questionnaire (HAq-II): psychometric properties. J Psychother Pract Res 5(3):260-271

Lutz W, Leon SC, Martinovich Z, Lyons JS, Stiles WB (2007) Therapist effects in outpatient psychotherapy: a three-level growth curve approach. JCouns Psychol 54(1):32-39. https://doi.org/10. 1037/0022-0167.54.1.32

Lutz W, Schiefele A-K, Wucherpfennig F, Rubel J, Stulz N (2016) Clinical effectiveness of cognitive behavioral therapy for depression in routine care: a propensity score based comparison between randomized controlled trials and clinical practice. J Affect Disord 189:150-158. https://doi.org/10.1016/j.jad.2015.08.072 
Maas CJ, Hox JJ (2005) Sufficient sample sizes for multilevel modeling. Methodology 1(3):86-92. https://doi.org/10.1027/1614-2241.1.3.86

Manes S, Nodop S, Altmann U, Gawlytta R, Dinger U, Dymel Wet al (2016) Social anxiety as a potential mediator of theassociation between attachment and depression. J Affect Disord 205:264-268. https://doi.org/10.1016/j.jad.2016.06.060

Mayo-Wilson E, Dias S, Mavranezouli I, Kew K, Clark DM, Ades A et al (2014) Psychological and pharmacological interventions for social anxiety disorder in adults: a systematic review and network meta-analysis. Lancet Psychiatry 1(5):368-376. https://doi.org/10.1016/S22150366(14)70329-3

Mogan R, Fischer R, Bulbulia JA (2017) To be in synchrony or not? A meta-analysis of synchrony's effects on behavior, perception, cognition and affect. J Exp Soc Psychol 72:13-20. https://doi. org/10.1016/j.jesp.2017.03.009

Mörtberg E (2014) Working alliance in individua and group cognitive therapy for social anxiety disorder. Psychiatry Res 220(1):716-718. https:// doi.org/10.1016/j.psychres.2014.07.004

Paulick J, Deisenhofer A-K, Ramseyer F, Tschacher W, Boyle K, Rubel J et al (2018) Nonverbal synchrony: a new approach to better understand psychotherapeutic processes and drop-out. J Psychother Integr 28(3):367-384. https://doi. org/10.1037/int0000099

Petzold MB, Bendau A, Ströhle A (2020) Körperliche Aktivität in der Prävention und Behandlung von Angsterkrankungen. Psychotherapeut. https:// doi.org/10.1007/s00278-020-00414-0

Ramseyer F, Tschacher W (2011) Nonverbal synchrony in psychotherapy: coordinated body movement reflects relationship quality and outcome. JConsult Clin Psychol 79(3):284-295. https://doi. org/10.1037/a0023419

Raudenbush SW, Bryk AS (2002) Hierarchical linear models. Applications and data analysis methods, 2. Aufl. SAGE, Thousand Oaks, London, New Dehli

Rief W, Schramm E, Strauß B (Hrsg) (2020) Psychotherapie-Ein kompetenzorientiertes Lehrbuch. Elsevier, München

Saxon D, Firth N, Barkham M (2017) The relationship between therapist effects and therapy delivery factors: therapy modality, dosage, and non-completion. Adm Policy Ment Health 44(5):705-715. https://doi.org/10.1007/s10488-016-0750-5

Schiefele A-K, Lutz W, Barkham M, Rubel J, Böhnke J, Delgadillo J et al (2017) Reliability of therapist effects in practice-based psychotherapy research: a guide for the planning of future studies. Adm Policy Ment Health 44(5):598-613. https://doi. org/10.1007/s10488-016-0736-3

Schoenherr D, Paulick J, Deisenhofer A-K, Schwartz B, Rubel J, Lutz W et al (2019a) Identification of movement synchrony: validation of time series analysis methods. PLoS ONE 14(2):e211494. https://doi.org/10.1371/journal.pone.0211494

Schoenherr D, Paulick J, Strauss B, Deisenhofer A-K, Schwartz B, Rubel J et al (2019b) Nonverbal synchrony predicts premature termination of psychotherapy for social phobic patients. Psychotherapy. https://doi.org/10.1037/ pst0000216

Schoenherr D, Paulick J, Worrack S, Strauss B, Rubel J, Schwartz B et al (2019c) Quantification of nonverbal synchrony using linear time series analysis methods: convergent validity of different methods. Behav Res Methods 51(1):361-383. https:// doi.org/10.3758/s13428-018-1139-z
Schoenherr D, Strauss B, Paulick J, Deisenhofer A-K Schwartz B, Rubel J et al (2019d) Movement synchrony and attachment related anxiety and avoidance in social anxiety disorder.J Psychother Integr. https://doi.org/10.1037/int0000187

Spitzer C, Masuhr O, Jaeger U, Brünig S, Dally A, FrickeNeef C et al (2015) Rolle der Bezugstherapeuten in der stationären Psychotherapie. Psychotherapeut 60(4):302-309. https://doi.org/10.1007/ s00278-015-0016-9

Stangier U, Clark DM, Ehlers A (2006) Soziale Phobie: Fortschritte der Psychotherapie Bd. 28 Hogrefe, Göttingen (Social phobia: Advances in psychotherapy)

Stekhoven DJ (2011) Using the missForest Package, S 1-11. https://stat.ethz.ch/education/ semesters/ss2012/ams/paper/missForest_1.2. pdf.Zugegriffen: 31. Aug. 2020

Wiltshire TJ, Philipsen JS, Trasmundi SB, Jensen TW, Steffensen SV (2020) Interpersonal coordination dynamics in psychotherapy: a systematic review. Cogn Ther Res. https://doi.org/10.1007/s10608020-10106-3

Woody SR, Adessky RS (2002) Therapeutic alliance, group cohesion, and homework compliance during cognitive-behavioral group treatment of social phobia. Behav Ther 33(1):5-27. https:// doi.org/10.1016/S0005-7894(02)80003-X

Zilcha-Mano S (2017) Is the alliance really therapeutic? Revisiting this question in light of recent methodological advances. Am Psychol 72(4):311-325 https://doi.org/10.1037/a0040435

Zuroff DC, Kelly AC, Leybman MJ, Blatt SJ, Wampold BE (2010) Between-therapist and withintherapist differences in the quality of the therapeutic relationship: effects on maladjustment and self-critical perfectionism. J Clin Psychol 66(7):681-697. https://doi.org/10.1002/jclp. 20683
Aktuelle Informationen

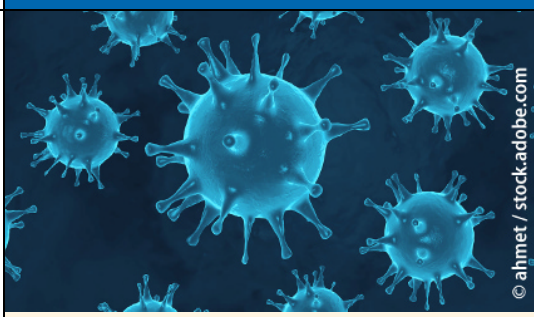

Aktuelle Entwicklung zu

COVID-19 bei Springer Nature und Springer Medizin

Springer Nature und Springer Medizin unterstützen die globale Reaktion auf die COVID-19-Pandemie, indem ein schneller und direkter $\mathrm{Zu}$ gang zu den neuesten verfügbaren Forschungsergebnissen und Daten ermöglicht wird.

Auf der Homepage SpringerMedizin.de finden Sie ein immer aktuelles Dossier mit Beiträgen, Forschungsarbeiten und Ergebnissen zu SARS-CoV-2 sowie relevanten Links.

Darin z.B. auch die kürzlich publizierte

Empfehlung von DIVI, DGIIN, DGAI und DGP zur Intensivtherapie von Patienten mit COVID-19.

Springer Nature arbeitet mit globalen Organisationen zusammen, und verlinkt über SpringerNature.com/de auf eine eigene Landingpage mit einer Vielzahl an Information sowie freiem Zugriff auf die COVID-19-Contentplattformen von Nature Research, BioMed Central (BMC) und Springer.

Das Dossier zu Coronavirus / Covid-19 von Springer Medizin finden Sie hier: www.springermedizin.de/covid-19

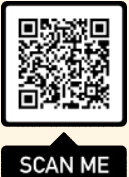

